\title{
Semantic effects in visual word detection with visual similarity controlled
}

\author{
LESLIE HENDERSON and JACKIE CHARD \\ The Hatfield Polytechnic, Hatfield, AL10 9AB, England
}

\begin{abstract}
In three experiments, subjects searched through word lists for a single target word. Search was faster when the target word belonged to a different semantic category from that of the background words with visual similarity controlled. This semantic effect increased with number of items to be searched through, and it obtained whether the target was cued visually or verbally. Semantic homogeneity within the background also speeded search, but only when subjects had no prior knowledge of the nature of a list. Several models of the semantic effect are described. All contrast the encoding of physical identity with the encoding of semantic attributes, but they differ in ascribing the effect to: (1) the relative access times for these codes, (2) the power of the codes in dealing with multiple comparisons, or (3) the attentional demands of comparison using different levels of code.
\end{abstract}

A traditional view of information processing in perception holds that feature analysis is organized into a sequence of levels that represents knowledge of increasing abstractness. Access to the system is via the most elementary level, and the flow of information is unidirectional. If, furthermore, processing terminates when a logically sufficient depth in the system has been reached and this information can be read off to control response decisions, then decision times will directly reflect the logical demands of the task. Neisser (1964) appeared to support this view with his observation that subjects in visual search tasks neither experience nor remember background items, as if these were examined only in sufficient depth to permit their rejection as targets.

Neisser's view was based on the study of letter search for small, fixed sets of targets, but even in this realm it is not without difficulties (see Henderson, 1978, for a review). Krueger (1970) has shown that subjects in visual search seem to be influenced by the sound of a letter's name, and Henderson (1973) has shown both facilitatory and inhibitory effects of letter names which are independent of visual similarity. These effects are compatible with the assumption that processing stages are ordered in abstractness only if it is also assumed that processing continues automatically to greater depth than is logically required by the nature of the decision.

Another kind of effect of category membership has been reported by Brand (1971), Ingling (1972), and Jonides and Gleitman (1972). They have demon-

This research was supported by SSRC Grant HR 3301 to the first author. A preliminary version of this paper was presented to the annual conference of the British Psychological Society, April, 1976. strated that search is faster when target and background items belong to different categories (letter vs. digit). In particular, it seems that the slope of the line relating detection time to number of comparisons required is diminished when background items belong to a different class from the target, as if the categorical difference somehow reduced the confusability of target with ground. Furthermore, Gleitman and Jonides (1976) and Jonides and Gleitman (1972) make the strong claim that the detection latency for the homographic character $O$ depends upon whether it is treated as "Oh" or "Zero" when among a given background category.

Isolated letters and digits are relatively simple visual stimuli, and they are members of a small set. It is not implausible, therefore, that the elementary feature analyzers could be directly connected to category feature lists, with the consequence that the distributions of access times for category information and for particular character identity overlap. When we turn to word stimuli and semantic category membership, this possibility is much less plausible. In the equivalent of Jonides and Gleitman's (1972) use of the character $O$, we might compare the search time for JAM as fruit conserve with JAM as traffic condition when embedded in a list of same or different class backgrounds. However, technically this is a very difficult experiment to control, so it is worth first attempting to manipulate background semantic class for a given word target.

Lawrence (1971) found that subjects could find a target defined as "an animal" more readily in a list of random background words than they could find a target defined as "nonanimal" in a list of animal words. Unfortunately, this study confounds the manner of defining the target (e.g., by negation) 
with the semantic homogeneity of the background items and with the semantic relationship of target to background.

Attempts to manipulate semantic relationships between target and background have recently been reported by Graboi (cited in Rumelhart, 1977) and by Karlin and Bower (1976). Graboi used a fixed target set of five words and highly practiced subjects, and found that search was slower when the background items belonged to the same semantic classes as the target words than when they were unrelated and heterogeneous. Karlin and Bower (1976) had subjects search through a fixed background class for a target set which in alternate blocks of trials belonged either to the same class as the target or to a different but homogeneous class. The existence of a semantic effect depended on target set size. For three- and sixword targets, but not for single-word targets, search was faster when target and background items belonged to a different semantic class. This effect increased in magnitude with increases in display size from two to six words.

These studies pose certain problems of interpretation. From Graboi's study, it is unclear whether the effect depends on the use of multiple targets. In addition, the target-to-background semantic relationship was confounded with the semantic homogeneity of the background itself. Karlin and Bower's study, on the other hand, maximized the likelihood that a relatively simple physical analysis can adjudicate between target and background. For example, their words were in lowercase print, which yields characteristic word envelopes, the words varied in number of letters, thereby providing an obvious lowlevel cue, and both target and background items were drawn from small, fixed sets.

The present experiments concentrate on the simple case of single-target searches. There are three main conditions of semantic relationships. In a same condition, targets are drawn from the same class as the background items. In a different condition, targets are drawn from a different class from the background which forms, nevertheless, a distinct homogeneous class. In a random condition, targets again are unrelated to background items, but these, in turn, are semantically unrelated to each other.

An attempt is made to minimize and to control the availability of gross physical distinctive features. Word length did not vary, and graphemic similarity of target to background was balanced across the semantic conditions. In the first two experiments, the subjects were instructed to search serially down a column of words, and in Experiment 3, the words were arranged in a clock display and the number of words in the display was varied.

\section{EXPERIMENT 1}

The first experiment consists of two parts, which are exact replications of each other conducted on different groups of subjects some months apart. The distinguishing feature of this experiment was that the target was verbally specified prior to each trial.

\section{Method}

Stimulus materials. The same stimulus materials were used in Experiments 1 and 2 . On each trial, a list of 12 words was searched for a single target word. Category norms were consulted in order to yield cohesive categories which allowed the selection of 2 target and 12 background items all with the same number of letters. Five such categories were used: four-legged animals, boys' names, girls' names, cities, and parts of the body, and all the words were of four letters. For each category, 2 of the 14 members were selected as targets. To be selected, they had to meet two criteria. They had to be high in production frequency, that is, be salient exemplars of the category; they also had to meet the specification that visual similarity between target and background items did not differ between the main semantic conditions. Target items were never used as background items in another trial.

The 2 targets from each of the five categories gave a total of 10 targets, which were used an equal number of times in each condition throughout the experiment. There were three semantic conditions which governed the nature of the list in which a target had to be searched for. In the same condition, the search was through a list containing background items drawn entirely from the target's own class (e.g., search for LION among ANIMALS). In the different condition, search was through a list of which the background items were drawn from a different, but still homogeneous, class from that of the target (e.g., search for LION among BOYS' NAMES). In the random condition, search was through a list of words unrelated to the target or to each other. For the purpose of the random condition, two further basic lists of 12 background items were constructed. This number of random lists was chosen so as to balance as closely as possible the frequency of occurrence of background items within the experiment. (Each of the two random lists was used 30 times, and each of the five categorized lists for the same and different conditions was used 24 times.)

Physical similarity of target to background was assessed by calculating the total number of occasions when a background word shared a letter in the same position as in the target. For first letters, sharing occurred a mean of 0.5 times per search list in each of the semantic conditions. For the remaining letter positions, this frequency was an average of 1.1 in the same and random conditions and 1.0 in the different condition.

An experimental session consisted of 180 trials, 60 in each of the three semantic conditions, with 40 being target-present and 20 target-absent trials. For the target-present condition, the eight list positions 2, 4, 6, 7, 8, 9, 10, 11 were used. Each position was used five times in each of the semantic conditions. These positions were divided randomly, but equally, between the two targets from a given semantic category so that LION, for instance, occurred at Positions 2, 6, 7, 10 and BEAR at Positions 4, 8, 9,11 . Insertion of a target in the list meant that one of the 12 possible background items was dropped. This was done at random for each display but having regard to the requirement to balance letter sharing. The order of the background items was randomized for each display.

Each 12-word display was typed in uppercase Pica type on a white card, with the words arrayed in a single-spaced vertical column. The words subtended a horizontal visual angle of $1.3^{\circ}$. The entire display subtended vertically $7.0^{\circ}$. 
The cards were presented in a two-field tachistoscope and were preceded and followed by a fixation field which indicated the location of the top of the word list.

Procedure. The subjects were instructed to respond as quickly and as accurately as possible. They were fitted with a throat microphone connected to a voice key, and its operation was explained. "Yes" and "no" responses indicated presence and absence of the target. The instructions emphasized the importance of fixating at the top of the list and searching down the list without recursions.

On each trial, the experimenter named the target and the subject, in his own time, fixated and depressed a trigger which replaced the fixation field with the word display for 3,000 msec and started the millisecond clock. Onset of the verbal response stopped the clock via the voice key. After 10 practice trials, each subject performed the 180 trials in a different order with all conditions randomized. Hence, subjects could not predict in advance what background condition would occur.

Subjects. In Experiment 1a, the 16 subjects consisted of 7 local sixth formers (grade 13), 7 undergraduates, and the 2 experimenters. In Experiment $1 \mathrm{~b}$, the 18 subjects were all undergraduates. All were paid volunteers.

\section{Results}

Analyses of variance were conducted separately on the target-present and target-absent RTs for each part ( $1 \mathrm{a}$ and $1 \mathrm{~b}$ ) of the experiment. All analyses contained semantic condition (same, different, random) as a factor and, in addition, the target-present analyses contained list position as an eight-level factor. Considering first yes responses (target present), we find a significant effect of list position in both parts of the experiment, $F(7,105$ and 7,119$)=144.2$ and $41.7, \mathrm{p}<.0001$. As would be expected, RT increased monotonically with position of the target in the list. Semantic condition also had a significant effect with the conditions ordered in both parts different, random, same in increasing order of $\mathrm{RT}, \mathrm{F}(2,30$ and $2,34)=3.5$ and 6.0 , ps $<.05$ and $<.01$. Using Tukey's test, differences of $42 \mathrm{msec}$ (a) and $38 \mathrm{msec}$ (b) are required for $\alpha=.01$. This indicated that different RTs were significantly faster than same RTs (59 and $70 \mathrm{msec}$ ) and significantly faster than random RTs (45 and $46 \mathrm{msec}$ ), whereas same and random $\mathrm{RTs}$ did not significantly differ (14 and $24 \mathrm{msec}$ ). The interaction between semantic condition and list position was also significant in both parts of the experiment, $F(14,210$ and 14,238$)=$ 3.3 and $2.4, \mathrm{p}<.005$.

Turning to the no RTs, we find again a significant effect of semantic condition, $F(2,30$ and 2,34$)=8.8$ and 9.2, p $<.005$. Differences of $67 \mathrm{msec}$ (a) and $60 \mathrm{msec}(\mathrm{b})$ are required for $\alpha=.01$. This indicated that different $\mathrm{RT}$ s were significantly faster than same RTs (143 and $141 \mathrm{msec}$ ). But they were significantly faster than random RTs only for part (b) of the experiment (17 and $70 \mathrm{msec})$. This time, random $\mathrm{RTs}$ were also significantly faster than same RTs (125 and $71 \mathrm{msec}$ ).

These data, with both parts of the experiment combined, are summarized in Figure 1, which shows

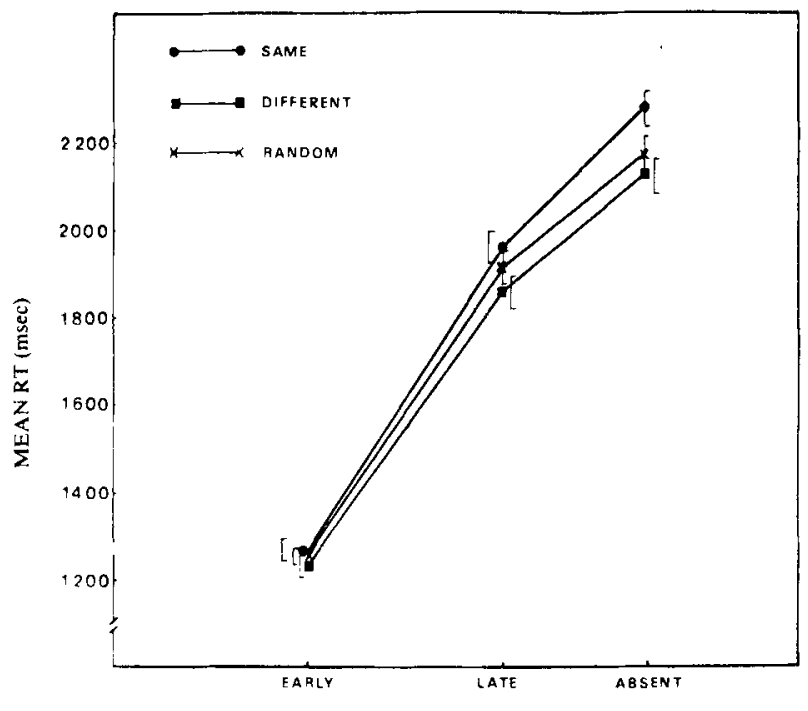

Figure 1. Reaction times for the three semantic conditions in Experiment 1 collapsed over parts a and $b$. The data are collapsed in to early positions (mean of Positions 2, 4, 6, 7), late positions (mean of Positions 8, 9, 10, 11), and target absent. Each of these categories contains an equal number of observations. Standard error is indicated by vertical bars.

reaction times for the three semantic conditions collapsed over the first four (early) and second four (late) list positions and for the target-absent condition. An equal number of observations contribute to each data point.

The error rate in both parts of the experiment was ordered over semantic conditions as follows: (1a) same $4.3 \%$, different $3.6 \%$, random $5.4 \%$; (1b) same $4.4 \%$, different $4.4 \%$, random $5.5 \%$. Analysis of variance revealed significantly more misses than falsealarm errors $(p<.001)$ but no significant effect of semantic conditions $(p>.10)$ or any interaction between type of response and semantic conditions $(\mathrm{p}>.10)$.

\section{EXPERIMENT 2}

It might be argued that the semantic effects found in Experiment 1 were a consequence of the verbal presentation of the target's depriving the subject of the opportunity to make a direct physical comparison of target with list items. On the other hand, if the target is introduced into the visual display to be searched, then its encoding incurs time costs, perhaps changing the strategy for encoding the target. In this experiment, therefore, the target was presented visually before each trial in the same physical form in which it occurred in the list.

\section{Method}

The materials and procedure were exactly as in Experiment 1 except that to specify the target before each trial the experimenter 
called out a number and the subject turned over a card bearing that number in order to reveal the target word. He then looked into the tachistoscope and initiated the trial in his own time.

Subjects. Nineteen paid undergraduate volunteers served as subjects. None had served in the previous experiment.

\section{Results}

The analysis of variance for the yes RTs once again revealed a significant effect of list position, $F(7,126)$ $=83.4, \mathrm{p}<.001$, and of semantic conditions, $F(2,36)=4.4, p<.05$. This time the interaction of these two factors just failed to achieve significance, $F(14,252)=1.7, p<.06$. Tukey's test required a minimum value of $41 \mathrm{msec}$ for $\alpha=.01$. This indicated that different $\mathrm{RT}$ s were significantly faster than same RTs $(65 \mathrm{msec})$ and significantly faster than random RTs $(50 \mathrm{msec})$ but random and same RTs did not differ significantly ( $24 \mathrm{msec})$.

For the no RTs, the effect of semantic conditions was again significant, $\mathrm{F}(2,36)=16.1, \mathrm{p}<.0001$, and comparisons indicated that different RTs were $188 \mathrm{msec}$ faster than same RTs $(\mathrm{p}<.01)$ and $51 \mathrm{msec}$ faster than random RTs $(\mathrm{p}<.05)$. Random RTs were, in turn, $137 \mathrm{msec}$ faster than same RTs $(\mathrm{p}<.01)$.

These results are summarized in Figure 2, which shows a highly similar general pattern to that in Experiment 1 .

The overall error percentages were ordered as in Experiment 1 (same 3.2\%, different $2.7 \%$, random $3.8 \%$ ). Analysis of variance on the error data showed significantly more errors on yes trials $(p<.0001)$ but no effect of semantic condition or any interaction between these factors $(p>.10)$.

\section{Discussion of Experiments 1 and 2}

It is clear that a semantic effect can be obtained when the target set size is one, contrary to the results

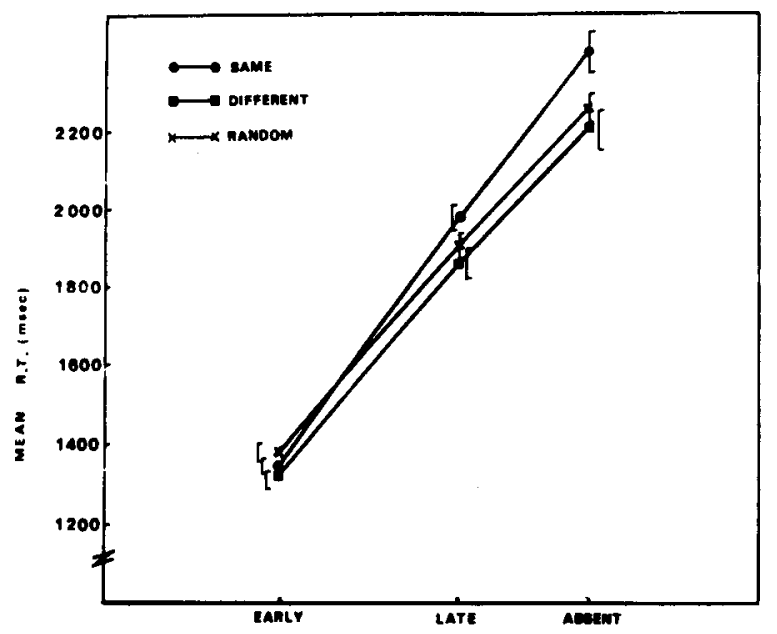

Figure 2. Reaction times for the three semantic conditions as a function of list position in Experiment 2. The data are collapsed over positions as in Figure 1.
Table 1

Mean No RTs in Experiments 1a, 1b, and 2 for Individual Target-Background Combinations in the Same and Different Conditions

\begin{tabular}{|c|c|c|c|c|c|c|}
\hline \multirow[b]{3}{*}{ Targets } & \multicolumn{6}{|c|}{ Background } \\
\hline & \multicolumn{2}{|c|}{$1 \mathrm{a}$} & \multicolumn{2}{|c|}{$1 \mathrm{~b}$} & \multicolumn{2}{|c|}{2} \\
\hline & $\mathrm{S}$ & $\mathrm{D}$ & $\mathrm{S}$ & $\mathrm{D}$ & $S$ & D \\
\hline LION & 2143 & 1998 & 2070 & 1935 & 2305 & 2017 \\
\hline BEAR & 2336 & 2126 & 2468 & 2096 & 2517 & 2101 \\
\hline LEGS & 2558 & 2041 & 2318 & 1939 & 2595 & 2128 \\
\hline FOOT & 2149 & 2141 & 2121 & 1978 & 2279 & 2193 \\
\hline BILL & 2231 & 2012 & 2130 & 1999 & 2288 & 2075 \\
\hline MIKE & 2345 & 2159 & $2249 *$ & 2378 & $2382^{*}$ & 2416 \\
\hline MARY & 2376 & 2318 & 2379 & 2207 & 2404 & 2256 \\
\hline BETH & $2120^{*}$ & 2201 & 2265 & 2206 & $2355^{*}$ & 2429 \\
\hline ROME & 2418 & 2318 & 2227 & 2097 & 2518 & 2202 \\
\hline YORK & 2251 & 2188 & 2217 & 2202 & 2336 & 2283 \\
\hline
\end{tabular}

same vs. different
$\mathrm{t}=$
2.78
2.93
3.23

*Indicates contrary direction of the effect.

of Karlin and Bower (1976). Moreover, another recent study has shown a semantic category effect with the use of a single target (Fletcher, Note 1, Experiment 3).

Given the emphasis we have placed on the control of nonsemantic relationships between target and ground, we conducted a post hoc examination of the RTs for individual target-background combinations. This analysis was conducted on the no RTs both because individual stimuli had been tagged in the analysis of noes and because variations in serial position and the replacement of one background item by the target would greatly complicate analysis of individual target-present stimuli. This analysis was directed toward two ends: first, an investigation of the reliability of the semantic effects over stimulus items, and second, an attempt to determine whether any of the variation in the magnitude of the semantic effect across stimuli was predictable from visual confusability.

Table 1 shows the RTs to individual search lists in Experiments $1 \mathrm{a}, 1 \mathrm{~b}$, and 2 for the critical comparison of the same and different conditions. From these data, it is clear that the semantic effect is reliable across stimuli.

Preliminary inspection suggested that those few stimuli for which the semantic effect was marginal or in the contrary direction were also ones in which the target bore a greater physical similarity to the different background. It will be remembered that letter-sharing was closely balanced across semantic conditions. However, this balance held for the average of the set of stimuli, whereas for individual pairs of stimuli letter sharing might favor one or the other condition. This allowed us to perform two analyses. In the first, we correlated RT with letter 
sharing for individual stimuli within each semantic condition. Any correlation obtained would necessarily be wholly independent of the semantic effects. For the second analysis, we considered pairs of stimuli having the same target, one from the same and one from the different background condition, correlating any difference between the pair, in amount of letter sharing with the difference in RT (e.g., the signed letter-sharing difference and signed RT difference between search for LION in a background of ANIMALS and of GIRLS). In this case, a high correlation would indicate that letter sharing and the semantic manipulation both affected RT with some variation in the magnitude of the semantic effect attributable to variation in letter sharing.

To compile an index of visual similarity for each stimulus, we totaled the number of letters shared in a given word position by target and background items, weighting leftmost letters by a factor of two. To obtain an RT estimate for each stimulus, we collapsed over the previous experiments plus an unreported experiment on 19 subjects which replicated Experiment 2 with instructions stressing speed. ${ }^{2}$ Thus, for 10 displays in each of the three semantic conditions, we obtained a mean RT based on 72 subjects.

The correlation of RT with visual similarity was positive within each semantic condition. It was highly significant within the same condition, tho $=.80$, $\mathrm{p}<.01$, but not significant for either the different, rho $=.54$ or the random condition, rho $=.30$. (While the variances of the visual similarity measures were approximately equal, the lower random condition correlation might have been partly attributable to the markedly lower RT variance for this condition.)

The magnitude of the RT effect between semantic conditions was also related to visual similarity. For the same vs. different comparison, the correlation was tho $=.79, \mathrm{p}<.01$, and for the random vs. different comparison the correlation was rho $=.66$, $\mathrm{p}<.05$. This tends to confirm that those few stimulus pairs for which the semantic effect is reversed are likely to be ones where the visual similarity on this occasion was greater in the different condition.

While this analysis is post hoc and is conducted over a limited range of low visual similarity values, it suggests that visual and semantic distinctiveness both affect detection times and that we get a clearer view of semantic effects when variation in visual similarity is taken into account. In particular, the semantic effects are likely to be obscured when gross physical cues such as word length and word envelope are available, as in Karlin and Bower (1976). But when these factors are minimized and controlled, a semantic effect is detectable even using a randomization of semantic conditions which prevents the subject from selecting a semantic strategy before his encounter with the display.
In summary, in each of Experiments $1 \mathrm{a}, 1 \mathrm{~b}$, and 2 , the overall effect of semantic relationships within the search list was significant both for yes and no responses, being invariably larger for no responses. The error rates, while not significantly influenced by the semantic conditions, were always ordered with different performance best and random performance worst.

Underlying the semantic conditions are two distinct comparisons, that between different and random, in which target is unrelated to background but the semantic homogeneity of the background is the critical variable, and that between different and same, in which the background is always semantically homogeneous but whether or not the target belongs to this semantic category is the critical variable.

The comparison of different with random. Of the six replications of this comparison across experiments and response types, all showed faster responding in the different condition, an effect which achieved significance in all but the target-absent condition in Experiment 1a. Figures 1 and 2 reveal no evidence that the effect enlarges at later list positions or for no responses, but rather it remains constant at about $40-60 \mathrm{msec}$. This tends to suggest that the effect of semantic homogeneity within background items is not located at the search stage, an issue to which we will return in the next experiment.

The comparison of different with same. In all the possible comparisons, performance in the different condition was significantly faster than in the same condition. The effect was generally about twice the size for no responses as for yes responses. Since no responses must depend upon exhaustive processing, this last finding suggests that the effect is located at the stage of analysis and comparison in which the background items are processed. However, an adequate test of slope differences between these conditions requires an experiment in which the number of elements in the display is varied, since in the present experiment interpretation of RT slopes over list position depends on unverifiable assumptions about the subject's scanning strategy.

\section{EXPERIMENT 3}

This experiment differed in three important respects from the previous ones. As we have noted, list position effects, even when backed by instructtions about search strategy, provide only a weak basis for estimating the RT slopes which reflect the search component of task performance. In the following experiment, therefore, we turn to the manipulation of set size, varying the number of words displayed in a clock-type format over the values 4 , 7 , and 10. 
A second change involved blocking rather than randomizing the semantic conditions so that subjects might preselect an appropriate strategy for a given relationship of target to background items.

Finally, a new set of stimulus material was generated by using new targets which were once again balanced across conditions for average amount of letter sharing. Given that we have shown search to be sensitive to the visual confusability of target with background, it might conceivably have been the case in the preceding experiments that the semantic relation of target to background had somehow become fortuitously confounded with some aspect of physical relatedness. In view of our control of letter sharing, any such confounding seemed unlikely. Nevertheless, by using a fresh set of target/background combinations, we could offer an independent test of the sufficiency of the semantic manipulation.

\section{Method}

Stimulus materials. Of the semantic categories used in the previous experiments, "cities" was dropped as having the lowest saliency members. From the remaining categories, the two least salient exemplars were dropped, as were two from each of the pair of random lists. The previously used targets were returned to the pool of background items and two new targets chosen, according to the rule that they be the highest items in production frequency for the category that satisfied the control of letter sharing. This yielded a total of eight targets, two from each of four semantic categories. Each of these was combined with a same, a different, and a random background to yield 24 combinations. These were factorially combined with three levels of set size $(4,7$, and 10 word displays) and with equiprobable presence or absence of the target for a total of 144 trials.

The stimulus displays were generated by computer. For each trial, the computer took the target item, when present, and a random selection from the background ensemble of 10 items according to the set size required. These were randomly allocated to points on a 10-position clock-type display, with the constraint, for set sizes of less than 10 , that not more than two adjacent positions be empty. This rule effectively insured that, with small set sizes, items were nevertheless distributed spatially so as to avoid easily fixated clusters. The words were typed in capitals by the Teletype onto white labels which were affixed to tachistoscope cards. These were presented to the subject exactly as in the previous experiments. Each word subtended a horizontal retinal angle of $1.3^{\circ}$. The entire display subtended a maximum angle of $6.1^{\circ}$ vertically and $7.0^{\circ}$ horizontally.

The results of the balancing of visual confusability across semantic conditions were as follows. Mean instances of first letter sharing between target and ground and letter sharing at all positions were, respectively, 0.37 and 0.71 for same, 0.62 and 0.68 for different, and 0.37 and 0.71 for random.

Procedure. The general procedure was as in the previous experiments. The subjects were provided with a list of the targets num bered 1 through 8 . These were in the same type as the search displays. On each trial, the target was specified by its number so that the subject had to inspect it visually using the target list. The subjects were instructed to fixate the center of where the clock display would appear, but no instructions were given about scanning strategy. Before each blocked semantic condition, the nature of the background items was explained with a sample display provided. The order of these blocks was counterbalanced across subjects. For each of the three blocks, the subject performed 10 practice trials followed by 48 experimental trials.
Subjects. Fifteen paid volunteer subjects were tested. None had taken part in the previous experiments. Twelve were secretarial staff and three were teaching staff.

\section{Results}

Mean correct RTs were entered into an analysis of variance, with order of semantic conditions as a between-subjects factor and semantic conditions (same, different, random), set size $(4,7,10)$, and response (yes, no) as within-subject factors. The main effect of order of testing did not approach significance; neither did any interaction in which it featured. All the other main effects were significant. Yes responses $(1,029 \mathrm{msec})$ were faster than no responses $(1,348 \mathrm{msec}), \mathrm{F}(1,12)=155.4, \mathrm{p}<.0001$. The overall effect of set size was significant, $F(2,24)$ $=200.0, \mathrm{p}<.0001$, with reaction times ordered, with increasing set size, 883, 1,196, 1,486 msec. Semantic conditions had a significant effect, $F(2,24)$ $=7.9, \mathrm{p}<.005$, with the RTs ordered different $(1,152 \mathrm{msec})$, random $(1,153 \mathrm{msec})$, same $(1,259 \mathrm{msec})$. Since the critical value for $\alpha=.01$ with Tukey's test is $58 \mathrm{msec}$, this indicated that both different and random RTs were significantly faster than same RTs, while not differing significantly from each other.

The only significant interactions were those of set size with response, $F(2,24)=54.7, p<.0001$, and set size with semantic conditions, $F(4,48)=3.2$, $\mathrm{p}<.05$. These are shown graphically in Figure 3, which displays both yes and no RTs for each of the semantic conditions as a function of set size, together with the best-fitting straight lines.

The intercept values were markedly larger for yes responses. This is consistent with the results of Karlin and Bower (1976) but contrary to the general tendency in binary classification tasks other than visual search. ${ }^{3}$ Comparison of pairs of semantic conditions within response types indicated that none of the intercept values differed significantly, $\mathrm{t}(14)_{\max }=1.55$.

Turning to the slope values, we find that these were generally half as great for yes and for no decisions, as might be expected in self-terminating search. The slope values for the different and random conditions did not differ significantly either for yes responses or for no responses, $t(14)<1$. The slope values for the same condition were about $20 \%$ greater than those for either the different or random condition. For no decisions, these differences were highly significant [S vs. $\mathrm{D}, \mathrm{t}(14)=2.71, \mathrm{p}<.01 ; \mathrm{S}$ vs. $\mathrm{R}$, $t(14)=4.56, p<.01]$, but for yes decisions, the differences failed to attain significance [S vs. $D, t(14)$ $=0.92$ n.s.; $\mathrm{S}$ vs. $\mathrm{R}, \mathrm{t}(14)=1.60, \mathrm{p}<.10]$. The differences were also significant when the yes and no 'data were combined, $t_{\min }=2.49, p<.02$. Again, there were overall more misses $(4.9 \%)$ than false alarms $(0.50 \%)$. The error rate was identical for the 


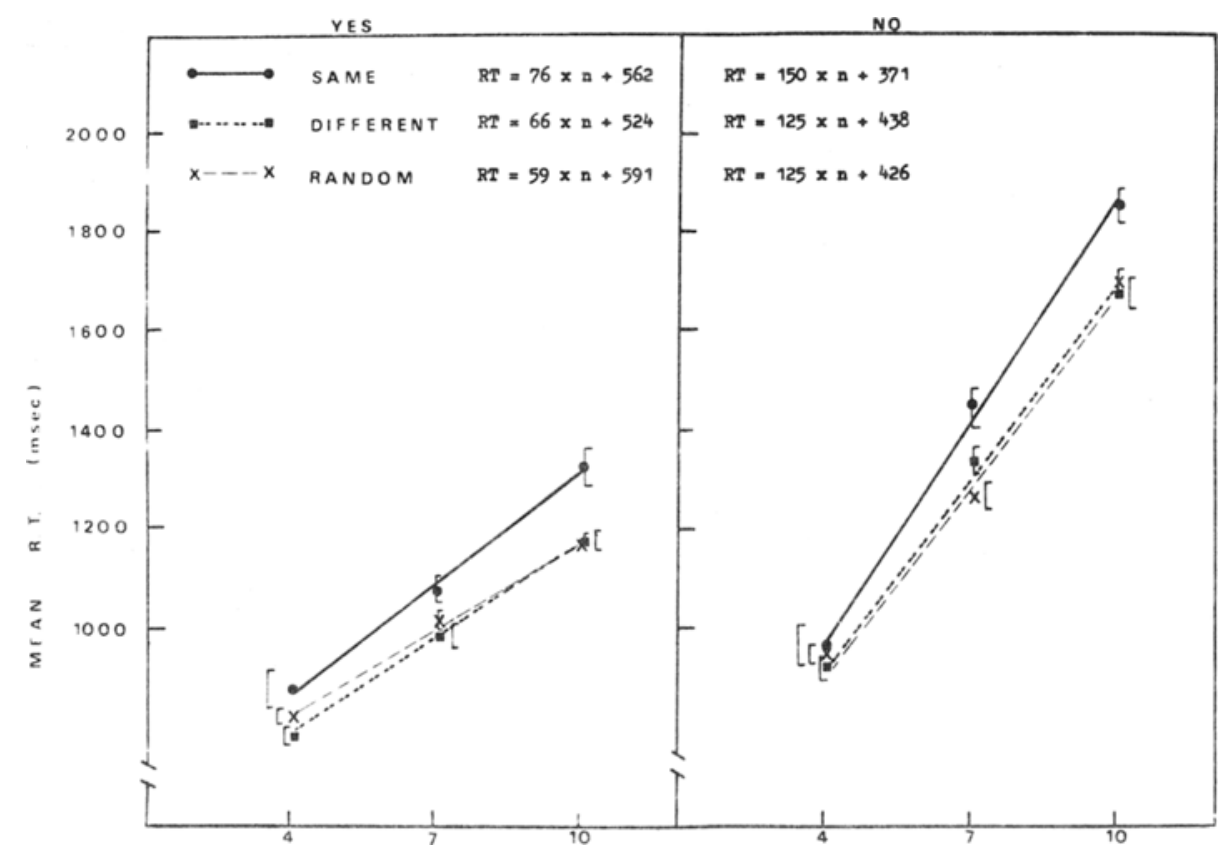

Figure 3. Reaction times for the three semantic conditions as a function of number of items in the search display for yes and no decisions. The best fitting straight lines are shown.

same and random conditions $(2.8 \%)$ and slightly lower for the different condition (2.6\%).

\section{Discussion}

These results may be summarized as indicating that the speed of the search process itself is greater when the target word is not drawn from the same category as the background words. Moreover, in this experiment, no additional advantage is attributable to semantic homogeneity within the background words.

Homogeneity within the background. The contrast between the advantage of different over random in the first two experiments and the lack of any such effect in the present experiment suggests that background homogeneity does not exert a fundamental influence on the search process. This suggestion is reinforced by the lack of any evidence that, when an effect does occur, it varies with number of items searched through.

It seems plausible that the disappearance of any effect in the present experiment was due to the change in a manipulation of the semantic conditions in blocks with full instructions to the subject about the nature of a forthcoming block. With conditions randomized, as in the earlier experiments, some extra, preliminary encoding time may have been spent in the random condition to appraise the nature of the lists. For example, a random list might require more sampling prior to directed search in order to determine that background items were unrelated semantically to the target.

Gibson (Note 2) also found no facilitation by background homogeneity when subjects were required to search for "an animal" either in a list of fruits or of random words. On the other hand, Fletcher (Note 1), using a design with no forewarning of conditions, did find small, but rather inconsistent, effects of homogeneity for targets defined by category as well as for specific word targets.

The lack of an effect of semantic homogeneity upon the search process is interesting for at least two reasons. First, it contrasts with the effect of associative priming frequently obtained in the lexical decision task (Meyer, Schvaneveldt, \& Ruddy, 1975), which even appears to hold when the priming word is presented subliminally (Marcel, Note 3 ). This suggests that while for priming to occur the primer does not require attention, the primed word does. Such attention is not paid to background items in search. Second, the lack of effect of homogeneity serves to preclude any theory which links it necessarily with the same vs. different effect in a general account based on distinctness in semantic space, where distinctness arose through the combined effect of the distance of target from ground and the compactness of target and background sets.

The categorical distinctiveness effect. The same vs. different effect was replicated using a fresh set of targets balanced for visual similarity and thus 
involving a fresh sample of physical relationships between target and background. This offers strong support to the claim that subjects can search more effectively when other members of the target's category do not appear as background distractors. The fact that this effect appears as a slope difference suggests that the effect is attributable to the speed with which background items are processed and rejected as candidate targets.

\section{GENERAL DISCUSSION}

A firm general conclusion which can be drawn from the effect of target-background semantic relationship in these experiments is that the hierarchical model with multiple readoff cannot be used to describe word search. However, it is not a simple matter to decide on the offending principle in that model. To an extent, we may classify competing theories of the semantic effect according to which principle of the traditional model they violate.

\section{Some Theoretical Alternatives}

One model, which has the merit of simplicity, has been advanced by Karlin and Bower (1976). They do not begin by challenging the traditional hierarchical model, but instead make the assumption that the same vs. different effect is essentially one which applies to multiple target searches. The superiority of the different condition arises, in their view, not through faster access to a semantic category code, but is due to the fact that this code, once derived, is a more powerful way of processing a multiple set of target possibilities which must otherwise be processed by serial exhaustive comparisons.

Responsibility for a category effect in Karlin and Bower's model rests with two sets of parameters. The first reflects the probability that a list will be processed in a categorical mode rather than by individual word search. The second parameters give the speed of processing in these two modes. On one hand, they estimate a parameter $c$, which describes the time taken in the categorical mode to classify a candidate list item and to compare it with the target category. On the other hand, they estimate $m$, the time taken in individual word search, to compare by exhaustive search the candidate item with the members of the target set. In their own experiment with a single target, $\mathrm{m}<\mathrm{c}$, and so it is efficient for their subjects to avoid the categorical mode. Our own repeated finding of a category effect with single targets suggests that it is the lack of control of visual features in their study which results in $m<c$. In particular, it is a consequence of their design that only in singletarget searches is word length a sufficient cue for response decisions. This notion appears to find further support in their observation that the value esti- mated for $m$ does not rise linearly with memory set size, as would be expected in serial exhaustive search. The exceptionally low value for set size of one could be due, they note, to a match of physical features which does not occur at larger target set sizes.

Furthermore, when Karlin and Bower attempt to generalize their account to cover the alphanumeric category effect, they are confronted with an effect which has been consistently obtained with single targets. They argue, however, that alphanumeric categorization can occur faster than the semantic categorization of words. Since they also assume, rather loosely, that identification time is the same for both types of material, they are able to conclude that alphanumeric stimuli can be categorized faster than they can be identified. It can easily be seen that this extension of their account to the single-target alphanumeric effect requires no appeal to the power of category codes for processing multiple target sets in parallel. In fact, it reduces to an encoding speed account of the sort that we consider below.

We turn, therefore, to the possibility, alluded to above, that the traditional heirarchical processing model incorrectly represents the process of encoding words. This might arise in one of two ways. The levels of the hierarchy might be connected up in counterintuitive ways such that elementary features are fed directly to semantic categorizers which can yield an output prior to that of identity analyzers. This structural possibility is the one advanced by Gleitman and Jonides (1976) with respect to the alphanumeric category effect. Its extension to word categories is nontrivial, however, in view not only of the number of categories but also of their multiple mappings and fuzzy boundaries. Such an account would have to encompass the visual confusability effect also obtained by assuming parallel processing of categorical and graphemic information with a "horse race" determining which process terminated first on a given trial.

An alternative encoding model violates, instead, the principle of bottom-up unidirectional flow of information and accommodates semantic effects by representing word encoding as an interactive process in which information about the higher order structure of words is used to support visual feature analysis. This sort of model has been examined by Henderson (1977) as an account of word superiority effects. Its extension to the category effect would require the assumption that the stores consulted by the feature analyzer are not purely lexical nodes but are organized with respect to semantic space. When background items excite neighboring regions in semantic space to that of the target, the feature analyzer demands more evidence before rejecting them as possibilities.

A final possibility is that the problem with the traditional model is the notion of multiple readoff, 
whereby, whenever a logically sufficient depth of processing is reached, information can be read off directly from that level to control response decisions. An alternative is that the effects of different amounts of stimulus structure do not arise due to encoding processes at all, but instead reflect subsequent control processes which govern comparison and decision processes.

An attentional control model has been advanced by Shiffrin and Schneider (1977) to account for perceptual learning in search performance. They distinguish between controlled search, which is slow, serial, demands attentional capacity, and is easily modified by the subject, and automatic detection, which is fast and parallel, difficult to alter, and is unaffected by load, needing attention only when the target is located. The main condition for automatic detection is that the target and background sets be disjoint throughout testing. Where this is so, categorization and automatic detection can be learned for arbitrarily constituted, visually confusable sets of characters. The role of predefined categorical distinctiveness is to improve controlled search and, possibly, to speed the acquisition of automatic detection.

It seems clear, however, that Shiffrin and Schneider's theory, like that of Karlin and Bower, is primarily intended to apply to multiple target searches and to the results of massive practice; they are therefore unable to derive unequivocal predictions about situations where categorical effects are manifest in a single experimental session with single targets (Shiffrin \& Schneider, 1977, p. 144, footnote 3).

If the advantage of categorical distinctiveness is that it requires less control of the search process and thus allows more comparisons to be handled in parallel, then this may account in part for the fact that the alphanumeric category effect is much larger than the present semantic effect with words and, in particular, the fact that alphanumeric different search exhibits a near zero slope of RT over set size. Alphanumeric displays are more compact and more items can be examined in a single fixation than is the case for word displays. Accordingly, there is more opportunity for an automatic search process to show its advantage.

These various theoretical possibilities that we have reviewed are quite vague about important details. Nevertheless, we believe, at the present stage, that it is more important to consider broad classes of theory which might be applied to a range of phenomena and paradigms, being clear about which general principles of the traditional model we are rejecting, than to pursue the ad hoc fit of a single model to a single experiment.
2. Gibson, E. J. The relationship between perceptual development and the acquisition of reading skill. Final report on Project No. 90046, U.S. Department of Health, Education and Welfare, 1971, pp. 30-37.

3. Marcel, A. J. Unconscious reading: Experiments on people who do not know that they are reading. Paper presented to the British Association for the Advancement of Science, September 1976. APU Research Report 1054-76, Cambridge.

\section{REFERENCES}

BRAND, J. Classification without identification in visual search. Quarterly Journal of Experimental Psychology, 1971, 23, 178-186.

Gleitman, H., \& Jonides, J. The cost of categorization in visual search: Incomplete processing of targets and field items. Perception \& Psychophysics, 1976, 20, 281-288.

Henderson, L. Effects of letter names on visual search. Cognitive Psychology, 1973, 5, 90-96.

Henderson, L. Word recognition. In N. S. Sutherland (Ed.), Tutorial essays in experimental psychology (Vol. 1). Hillsdale, N.J: Erlbaum, 1977.

Henderson, L. Pandemonium and visual search. Perception, 1978, in press.

INGLING, N. W. Categorization: A mechanism for rapid information processing. Journal of Experimental Psychology, 1972, 94, 239-243.

Jonides, J., \& Gleitman, H. A conceptual category effect in visual search: $O$ as a letter or a digit. Perception \& Psychophysics, 1972, 12, 457-460.

KarLin, M. B., \& Bower, G. H. Semantic category effects in visual search. Perception \& Psychophysics, 1976, 19, 417-424.

KRUEGER, L. E. The effect of acoustic confusability on visual search. American Journal of Psychology, 1970, 83, 389-400.

LAWRENCE, D. H. Two studies of visual search for word targets with controlled rates of presentation. Perception \& Psychophysics, 1971, 10, 85-89.

Meyer, D. E., Schvaneveldt, R. W., \& Ruddy, M. G. Loci of contextual effects on visual word-recognition. In P. M. A. Rabbitt \& S. Dornic (Eds.), Attention and performance $V$. London: Academic Press, 1975. Pp. 98-118.

NeIsser, U. Visual search. Scientific American, 1964, 210, 94-102.

Rumelhart, D. E. Toward an interactive model of reading. In S. Dornic \& P. M. A. Rabbitt (Eds.), Attention and performance VI. New York: Academic Press, 1977.

Shiffrin, R. M., \& Schneider, W. Controlled and automatic human information processing: II. Perceptual learning, automatic attending and a general theory. Psychological Review, 1977, 84, 127-190.

\section{NOTES}

1. A list of the stimulus material can be obtained from the authors.

2. This experiment used the same stimuli and procedure as Experiment 2, save for the "risky" criterion instructions. The same vs. different effect was reliable, though reduced in magnitude.

3. This extra time taken for yes responses is probably attributable to the attentional demand of noting the target when it is encountered in the list.

\section{REFERENCE NOTES}

1. Fletcher, B. Semantic effects on non target and target decision times in a classifi : tion task. Unpublished manuscript, 1977.
(Received for publication October 10, 1977; revision accepted November 30, 1977.) 